of the American Thoracic Society and Infectious Diseases Society of America. Am J Respir Crit Care Med 2019;200(7):e45-e67.

9. GOLD Global Strategy for the Diagnosis, Management and Prevention of COPD. Global Initiative for Chronic Obstructive Lung Disease (GOLD) website. https://goldcopd.org/wp-content/uploads/2017/11/GOLD-2018-
v6.0-FINAL-revised-20-Nov_WMS.pdf. Published 2018. Accessed November 29, 2020.

10. Vandenberg AE, Vaughan CP, Stevens $M$, et al. Improving geriatric prescribing in the ED: a qualitative study of facilitators and barriers to clinical decision support tool use. Int J Qual Health Care 2017 Feb 1;29(1):117-123.

\title{
Can intravenous antimicrobial start data reported to the National Healthcare Safety Network determine appropriateness of antibiotic use in hemodialysis patients?
}

\author{
Priti R. Patel MD, MPH ${ }^{1}$, Shannon A. Novosad MD, $\mathrm{MPH}^{1}$ and Ibironke W. Apata $\mathrm{MD}^{1,2}$ \\ ${ }^{1}$ Centers for Disease Control and Prevention, Atlanta, Georgia and ${ }^{2}$ Emory University School of Medicine, Atlanta, Georgia
}

To the Editor-In their article, "Inappropriate intravenous antimicrobial starts: An antimicrobial stewardship metric for hemodialysis facilities," Hahn et al $^{1}$ describe their application of data reported to CDC's National Healthcare Safety Network (NHSN) to determine appropriateness of IV antibiotic use in outpatient hemodialysis centers. NHSN's Dialysis Event (DE) surveillance system was designed to track bloodstream infections (BSIs) and other vascular access infections in hemodialysis outpatients through monitoring of events such as positive blood cultures. The authors examined outpatient IV antimicrobial start (IVAS) events reported to NHSN and considered any IVAS without documentation of coreported positive blood culture, collection of blood sample for culture, or local access site infection to be inappropriate, even when symptoms such as fever, chills, rigors, or drop in blood pressure were present. We applaud these investigators for drawing necessary attention to the issue of antibiotic use in dialysis patients, which is an important area of study with limited data, and for exploring the use of data to inform improvement in practice. However, we have concerns about their approach to the categorization of antibiotic use without incorporation of relevant clinical information or validation of NHSN data for this purpose, and the potential for unintended consequences among patients at high risk for infections and sepsis.

Intravenous antimicrobial starts largely represent empiric antibiotic doses for suspected infection. Initiating empiric antibiotics in the presence of signs and symptoms of serious infection, while awaiting culture information, should be considered appropriate use. ${ }^{2}$ However, Hahn et al. excluded symptom data from their assessment of appropriateness, even for the 260 IVAS events (27\%) for which these data were available in NHSN. They explained that the data were not consistently reported. We agree that gaps exist in the completeness of the symptom information, which is one of several inherent limitations with the use of NHSN data in this manner. The IVAS events that occurred in the context of symptoms but without preceding blood culture collection may represent gaps in documentation (of blood cultures) or adherence to blood culture collection protocols, rather than actual inappropriate antibiotic use. A more robust appropriateness

\footnotetext{
Author for correspondence: Priti Patel, E-mail: ppatel@cdc.gov

Cite this article: Patel PR, Novosad SA, and Apata IW. (2022). Can intravenous antimicrobial start data reported to the National Healthcare Safety Network determine appropriateness of antibiotic use in hemodialysis patients?. Infection Control \& Hospital Epidemiology, 43: 674-675, https://doi.org/10.1017/ice.2021.55
}

determination should incorporate medical record review to identify symptoms that might have been unreported to NHSN, and other relevant information.

Hahn et al acknowledge that they did not attempt to identify related events in the same patient (that might justify the IVAS) if they were not coreported on the same form. The NHSN does not require that events such as positive blood cultures and IVAS be coreported as long as each event is reported. Unique identifiers exist within NHSN to facilitate linkage of events that occurred in the same patient, even when events are not submitted together. ${ }^{3}$ Other potential justifications for antibiotic administration, such as surgical prophylaxis (for which blood culture collection is not typically warranted prior to antibiotic administration), are not captured within the dialysis event surveillance; thus, they were not factored into the appropriateness determination.

Previous studies have identified de-escalation of antibiotic therapy as the most common missed opportunity for improved antibiotic use among patients on hemodialysis, ${ }^{4,5}$ suggesting that continued antibiotic doses, as opposed to the initial dose, should be the priority for antibiotic use measurement efforts in this setting. However, the NHSN does not capture all IV antibiotic doses administered to dialysis patients, nor the specific antibiotic administered (other than vancomycin starts). In our opinion, expanded efforts are needed to identify stewardship interventions that could result in meaningful improvements in outcomes in this population, to help guide the development of useful metrics.

Hahn et al conclude that $57.5 \%$ of all IVAS in the facilities examined were inappropriate. Given the aforementioned challenges, we believe that they may have overestimated true inappropriate prescribing of IV antibiotics in dialysis facilities. Prior studies have demonstrated that initial doses of antibiotic treatment are a small proportion of all antibiotic doses received by hemodialysis outpatients (eg, 7.6\% in one study). ${ }^{4,5}$ In previous studies of maintenance hemodialysis patients, $10 \%-43 \%$ of initial IV antibiotic treatment doses were classified as inappropriate, ${ }^{4,5}$ and $20 \%-30 \%$ of all vancomycin or IV antibiotic doses prescribed (including continued doses) were classified as inappropriate. ${ }^{5,6}$ Few studies have evaluated antibiotic use in outpatient hemodialysis centers, and the resultant estimates of inappropriate use are often challenged by limited medical record documentation. ${ }^{4,5,7}$ Hahn et al also draw parallels between outpatient hemodialysis care and outpatient primary care settings. However, hemodialysis clinics and patients are unlike most general outpatient 
care settings and populations. Bloodstream infections and resultant complications are common among hemodialysis patients; treating suspected infections and sepsis early is essential to quality care.

Notable strengths of the Hahn et al study include the examination of details associated with antibiotic starts on a larger scale than previous studies, including 1 year of data from $\sim 50$ dialysis facilities across Philadelphia County. The authors additionally demonstrated that collection of blood cultures prior to initiating empiric treatment for suspected bloodstream infections may be an area for improved antibiotic prescribing - a finding consistent with previous studies in this setting. ${ }^{4,5,7}$ Collection of blood cultures prior to initiating antibiotics for suspected bloodstream infections is integral to ensuring appropriate antibiotic selection and has the potential of improving infection cure rates. ${ }^{2,7}$

Antibiotic stewardship involves strategies to promote the optimal use of antibiotics. ${ }^{7}$ In the hemodialysis patient population, optimal use includes administration of empiric antibiotics to treat documented and suspected infections early and tailoring antibiotics based on culture results. Although routinely collected metrics for antibiotic use should be easily obtainable, oversimplified measures of appropriateness can potentially undermine the goals of stewardship and lead to misdirection of scarce resources. Expanding upon information captured through NHSN and validating new applications of the data might help to advance the science surrounding stewardship in outpatient dialysis settings. Additional, promising areas for intervention in this setting include standardization of blood culturing practices, improved communication among providers and across care transitions, and strengthened infection prevention programs and implementation. ${ }^{7}$
Acknowledgments. The findings and conclusions in this report are those of the authors and do not necessarily represent the official position of the Centers for Disease Control and Prevention.

Financial support. No financial support was provided relevant to this article.

Conflicts of interest. All authors report no conflicts of interest relevant to this article.

\section{References}

1. Hahn PD, Figgatt M, Peritz T, Coffin SE. Inappropriate intravenous antimicrobial starts: An antimicrobial stewardship metric for hemodialysis facilities. Infect Control Hosp Epidemiol 2019;40:1178-1180.

2. Standardization of blood culture collection for patients receiving in-center hemodialysis. Nephrologists Transforming Dialysis Safety website. https://www.asn-online.org/g/blast/files/

NTDS_Blood_Culture_Collection_Standardization_combined_01.16.2020. pdf. Published January 2020. Accessed February 8, 2021.

3. Dialysis event surveillance protocol. Centers for Disease Control and Prevention website. https://www.cdc.gov/nhsn/pdfs/pscmanual/8pscdialysis eventcurrent.pdf. Published February 2018. Accessed February 8, 2021.

4. Snyder GM, Patel PR, Kallen AJ, Strom JA, Tucker JK, D’Agata EM. Antimicrobial use in outpatient hemodialysis units. Infect Control Hosp Epidemiol 2013;34:349-357.

5. Zvonar R, Natarajan S, Edwards C, Roth V. Assessment of vancomycin use in chronic haemodialysis patients: room for improvement. Nephrol Dial Transplant 2008;23:3690-3695.

6. Green K, Schulman G, Haas DW, Schaffner W, D’Agata, EM. vancomycin prescribing practices in hospitalized chronic hemodialysis patients. Am J Kidney Dis 2000;35:64-68.

7. Apata IW, Kabbani S, Neu AM, et al. Opportunities to improve antibiotic prescribing in outpatient hemodialysis facilities. Am J Kidney Dis 2020. doi: 10.1053/j.ajkd.2020.08.011.

\title{
Time to remind us that absence of evidence is not evidence of absence during the coronavirus disease 2019 (COVID-19) pandemic
}

\author{
Chenyu Sun MD, MSc ${ }^{1}$ (1), Ce Cheng $\mathrm{DO}^{2}$ (1), Mubashir Ayaz Ahmed MD ${ }^{1}$ (1) and Qin Zhou $\mathrm{PhD}^{3}$ (1) \\ ${ }^{1}$ AMITA Health Saint Joseph Hospital Chicago, Chicago, Illinois, ${ }^{2}$ The University of Arizona College of Medicine at South Campus, Tucson, Arizona and ${ }^{3}$ Radiation \\ Oncology, Mayo Clinic, Rochester, Minnesota
}

To the Editor-During the current coronavirus disease 2019 (COVID-19) pandemic, guidelines issued by various agencies, including the US Centers for Disease Control and Prevention (CDC), have been conflicting on the issue of respiratory protection with a face mask or a respirator. ${ }^{1}$ The CDC has not officially announced the protective effects of masks for the wearers until recently, ${ }^{2}$ and even now, its web pages still show that surgical mask "is not considered respiratory protection." 3

\footnotetext{
Author for correspondence: Chenyu Sun, E-mail: drsunchenyu@yeah.net

Cite this article: Sun C, et al. (2022). Time to remind us that absence of evidence is not evidence of absence during the coronavirus disease 2019 (COVID-19) pandemic. Infection Control \& Hospital Epidemiology, 43: 675-676, https://doi.org/10.1017/ice.2021.47
}

Earlier this year, research on the protective effects of masks was limited, indicating lack of sufficient evidence to support the protective effects of masks to severe acute respiratory syndrome coronavirus 2 (SAS-CoV-2). However, the lack of evidence that masks have protective effects to respiratory viral infections is not equivalent to evidence that masks lack protective effects. It would be prudent to refrain from premature conclusions without further comprehensive studies. As Mark Twain said, "It ain't what you don't know that gets you into trouble. It's what you know for sure that just ain't so." At this time, we remind ourselves that the absence of evidence is not evidence of absence. ${ }^{4}$ Interestingly, while evidence of mask use against other viruses has not been strong enough for the CDC to suggest the protective effects of mask wearing, ${ }^{5}$ remdesivir was approved for emergency or experimental use, with only limited evidence, as a therapeutic candidate due to its ability to inhibit SARS-CoV-2 in vitro and against other 